\title{
High-efficiency siRNA-based gene knockdown in human embryonic stem cells
}

\author{
YINGHONG MA, ${ }^{1}$ JIANYU JIN, ${ }^{2,3}$ CHUNSHENG DONG, ${ }^{1}$ EE-CHUN CHENG, ${ }^{1}$ HAIFAN LIN, ${ }^{1}$ \\ YINGQUN HUANG, ${ }^{1,2}$ and CAIHONG QIU ${ }^{\mathbf{1}}$ \\ ${ }^{1}$ Yale Stem Cell Center, New Haven, Connecticut 06520, USA \\ ${ }^{2}$ Department of Obstetrics, Gynecology, and Reproductive Sciences, Yale University School of Medicine, New Haven, Connecticut 06510, USA \\ ${ }^{3}$ Wenzhou University College of Teacher Education, Wenzhou, Zhejiang 325035, China
}

\begin{abstract}
Loss-of-function studies in human embryonic stem cells (hESCs) and induced pluripotent stem cells (iPSCs) via nonviral approaches have been largely unsuccessful. Here we report a simple and cost-effective method for high-efficiency delivery of plasmids and siRNAs into hESCs and iPSCs. Using this method for siRNA delivery, we achieve $>90 \%$ reduction in the expression of the stem cell factors Oct4 and Lin28, and observe cell morphological and staining pattern changes, characteristics of hESC differentiation, as a result of Oct4 knockdown.
\end{abstract}

Keywords: stem cells; siRNA; transfection

\section{INTRODUCTION}

Human embryonic stem cells (hESCs) are capable of selfrenewal and differentiation into all derivatives of three primary germ layers, giving rise to every cell in the human body (Thomson et al. 1998). These features render hESCs a valuable model system for studying human embryogenesis, dissecting mechanism of human diseases, screening new drugs, and engineering tissues or even organs. Induced pluripotent stem cells (iPSCs) are believed to be essentially identical to hESCs and also bear great therapeutic potential since they can be generated from patients directly (Takahashi et al. 2007; Yu et al. 2007). To capitalize on the potential of hESCs and iPSCs, it is imperative to have an effective method to introduce genes into these cells or to knock down the expression of specific genes for genetic dissection of the molecular mechanisms underlying their proliferation and differentiation. RNA interference technology for loss-of-function studies is a powerful tool for these purposes.

Reprint requests to: Yingqun Huang, Yale Stem Cell Center, PO Box 208073, New Haven, CT 06520, USA, or Department of Obstetrics, Gynecology, and Reproductive Sciences, Yale University School of Medicine, New Haven, CT 06510, USA; e-mail: yingqun.huang@yale.edu; fax: (203) 785-7134; or Caihong Qiu, Yale Stem Cell Center, PO Box 208073, New Haven, CT 06520, USA; e-mail: caihong.qiu@yale.edu; fax: (203) 785-4305.

Article published online ahead of print. Article and publication date are at http://www.rnajournal.org/cgi/doi/10.1261/rna.2350710.
Transient delivery of siRNA duplexes into cells facilitated by cationic lipid carriers such as Lipofectamine 2000 has been widely used for gene targeting in mammalian cells. However, this regime does not work well with hESCs, likely, in part, due to the low cloning (growing cell colonies from single cells) efficiency of hESCs. In most studies, hESCs are manipulated as small cell clumps, but not single cells, since single hESCs generated by enzymatic digestion suffer from low cell viability (Zaehres et al. 2005). This not only makes the process of clonal selection difficult, but also causes inaccessibility of the inner cells for transfection materials and reagents. In addition, the overgrowth of untransfected cells may overwhelm and dilute transfected cells, further reducing transfection efficiency. These limitations make hESC high transfection efficiency difficult to reach and high-efficiency siRNA-mediated gene knockdown hard to attain. To circumvent these problems, lentivirus delivery has been developed to target genes in hESCs (Zaehres et al. 2005). Although highly efficient transduction and gene knockdown have been achieved using this system, the process involves potential challenges in virus construction, packaging, and obtaining a sufficiently high titer, as well as the copy number and position effect of virus insertion into the genome. Here, we report a method that combines the usage of Accutase and ROCK inhibitors with single cell suspension transfection using Lipofectamine 2000 as a delivery reagent to achieve a $>90 \%$ efficiency in transient silencing of endogenous Lin 28 and Oct4 genes in both hESCs and iPSCs. 


\section{RESULTS}

We have previously reported that preincubation of single cell suspension in transfection cocktails can dramatically increase transfection efficiency (Zhang et al. 2007). However, this strategy did not work well with hESCs, mainly due to their low viability in single cell suspension. We therefore initiated efforts to increase cell viability using cloning efficiency as a read-out. First, we used hESCs that were grown in Matrigel-coated plates under feeder-free conditions. Under these conditions, the cells were maintained as single layers that were easily dissociated into single cells (Fig. 1A,B). Second, we used Accutase (a mixture of proteolytic and collagenolytic enzymes) to dissociate the cells. It has been reported that using Accutase instead of trypsin can significantly improve cell viability (Bajpai et al. 2008). Third, we treated cells with a ROCK (Rho-associated kinase) inhibitor before and during transfection. ROCK inhibitors significantly increase the viability of hESCs during passaging, cryopreservation, and thawing, without adversely affecting their self-renewal or differentiation (Claassen et al. 2009; Watanabe et al. 2007). Combining the above three approaches, we were able to drastically increase the cloning efficiency. We obtained $1775 \pm 305$ colonies from a single cell suspension containing $1 \times 10^{5}$ cells, while only $7 \pm 1$ colonies were obtained from cells grown on feeders, dissociated by trypsin, and without the treatment with the ROCK inhibitor. Figure 1, C and D, shows representative images of cell colonies resulting from the different methods.

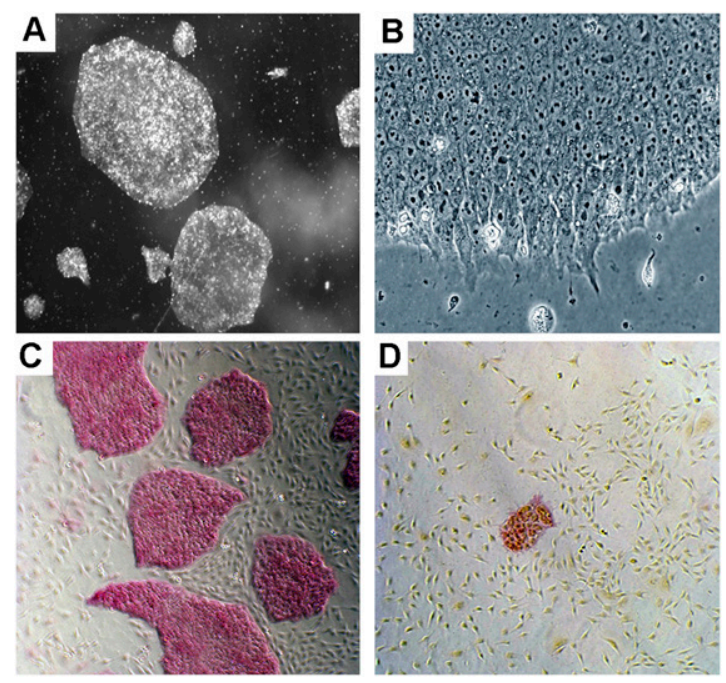

FIGURE 1. Colony and cell morphology of hESCs grown under feeder-free, serum-free, and components-defined conditions. $(A, B)$ Images of cell colonies taken at $20 \times$ and $200 \times$ magnifications, respectively. $(C, D)$ Images of cell colonies resulted from cloning assays using our method and the traditional method, respectively. The pink color indicates undifferentiated alkaline phosphatase (AP) positive cells. Images were taken at a $40 \times$ magnification.
As a proof of principle that transfection in single cell suspension can, in general, augment the transfection efficiency in hESCs, we tested a GFP reporter plasmid (pSUPERGFP) in H1 cells. Thirty-six hours after the transfection, cells were subjected to immunofluorescence and fluoresenceactivated cell sorting (FACS). By immunofluorescence, the majority of growing cells were GFP positive (Fig. 2A). FACS analysis revealed that GFP-positive cells accounted for $69.8 \%$ of the population (Fig. 2B). In general, as we recover nearly $90 \%$ of viable cells $24 \mathrm{~h}$ after the transfection (data not shown), we conclude that this method is associated with low cytotoxicity. It is worthwhile to point out here that although a transfection efficiency of $50 \%-60 \%$ has been reported for hESCs (Hohenstein et al. 2008), this alternative method requires limited cell numbers, nucleoporators, and specialized reagents, which is in contrast to ours, where no special equipment or reagents are involved.

Inspired by the above observations, we next set out to test siRNAs in $\mathrm{H} 1$ cells. First, we used a siRNA specific for Lin28 (siLin28) (Qiu et al. 2010). Lin28 is an RNA-binding protein that is highly expressed in undifferentiated hESCs and is among four factors (including Oct4, Sox2, and Nanog) used for making iPSCs (Yu et al. 2007). Using the standard transfection method, we obtained $\sim 50 \%$ knockdown efficiency on Lin 28 at the RNA level $72 \mathrm{~h}$ following siLin 28 transfection, compared with control siRNA (siCon) transfection (Fig. 3A, cf. light bars with dark bars). In contrast, we were able to reduce Lin 28 expression by $77 \%$ at the RNA level $24 \mathrm{~h}$ after transfecting the same siRNA, while as expected, the untargeted $\beta$-actin mRNA was not affected (Fig. 3B, cf. gray bars with black bars). At the protein level, there was a $6 \%$ decrease at the 24 -h time point (Fig. 3C, bottom blot, cf. lane 2 with lane 1$)$. When samples were collected and analyzed $72 \mathrm{~h}$ after siRNA transfection, even greater knockdown efficiencies were obtained: $98 \%$ at the RNA level (Fig. 3D) and essentially 100\% at the protein level (Fig. 3E). In this particular experiment (Fig. 3D,E), a second siRNA transfection (double transfection) was conducted $48 \mathrm{~h}$ following the first siRNA transfection, and samples were collected and analyzed $24 \mathrm{~h}$ after the second transfection. Although in the case of siLin 28 a single transfection is often sufficient to achieve a $>90 \%$ knockdown (data not shown), double transfection ensures a $>95 \%$ efficiency.

To determine whether this method is also effective for silencing other genes, we tested a siRNA specific for Oct4, a transcription factor that is crucial for ESC self-renewal and pluripotency (Pei 2009). We observed a 59\% and 69\% decrease in Oct4 expression at the RNA and protein level, respectively, $24 \mathrm{~h}$ after a single transfection (Fig. 4A,B). Double transfection increased the efficiency to $90 \%$ and $80 \%$ at the RNA and protein levels, respectively (Fig. 4C,D), and the knockdown level remained low for at least $6 \mathrm{~d}$ following the initial transfection (Fig. 4E,F).

To ask whether this method also works in other hESC lines, we used the $\mathrm{H} 7$ line, a female embryo-derived line 

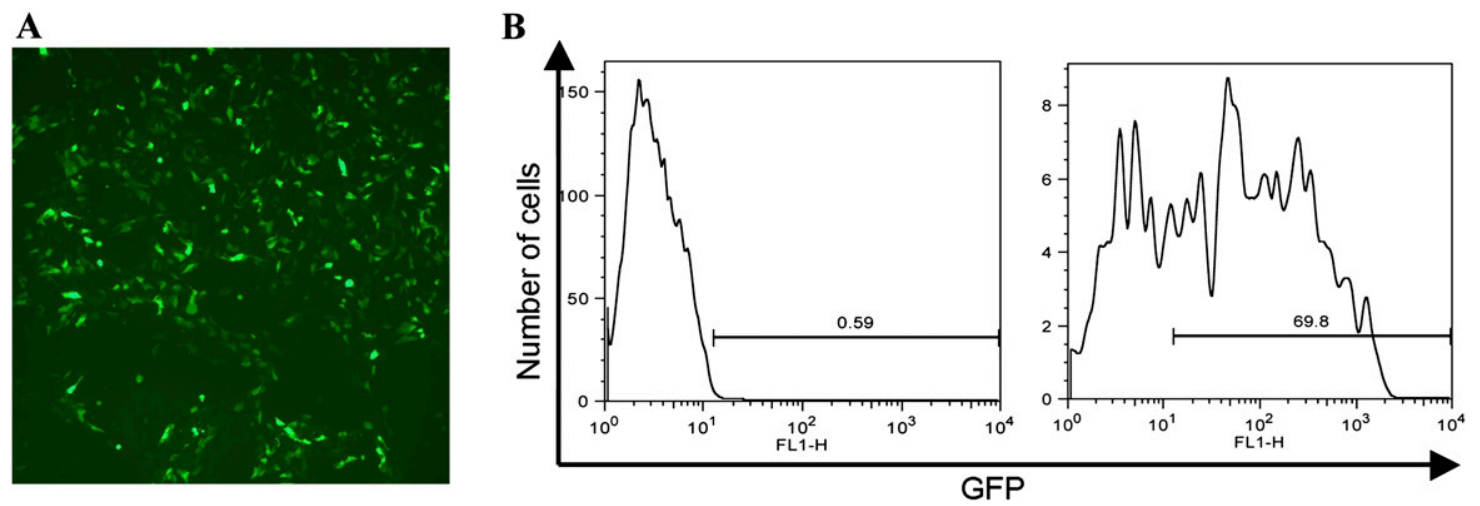

FIGURE 2. (A) Immunofluorescence of $\mathrm{H} 1$ cells $36 \mathrm{~h}$ after transfection with a GFP reporter plasmid. (B) Results of FACS analysis of H1 cells mock transfected (left) or transfected with the GFP reporter plasmid (right).

(H1 cells were derived from a male embryo). We observed a $90 \%$ and $72 \%$ knockdown of Oct 4 at the RNA and protein level, respectively (Fig. 4G,H).

To determine whether Oct4 knockdown leads to cell differentiation, we performed morphologic analysis. We initially noticed morphological changes within $72 \mathrm{~h}$ following transfection (data not shown), and the changes became more prominent at day 6 after the first transfection. As shown in Figure 5B, cells located at the center of the colony exhibited enlarged cell size, increased cytoplasmic area, and a decreased nuclear-to-cytoplasmic ratio, characteristic of differentiated cells. In contrast, no morphological changes were detected in the control siRNA-transfected cells (Fig. $5 \mathrm{~A})$. To provide further evidence for cell differentiation, we examined the expression of alkaline phosphatase (AP), which is expressed in undifferentiated hESCs. Six days after the first transfection of siOct4, most cells at the center of the colonies lost staining for AP (Fig. 5D), while those around the edges of the colonies remained AP positive (Fig.
5D). In contrast, cells transfected with control siRNA remained AP positive throughout the colonies (Fig. 5C). Thus, siOct 4 transfection leads to both cell morphological change and loss of AP staining, consistent with hESC differentiation.

Finally, we tested this method in iPSCs. Human iPSCs first generated in 2007 (Takahashi et al. 2007; Yu et al. 2007) are of great therapeutic potential owning to their patient specificity. The iPSC line we used was derived from reprogramming of human fibroblasts using the Yamanaka factors (Takahashi et al. 2007). We tested silencing of endogenous Lin28 using siLin28. As shown in Figure 3, $\mathrm{F}$ and $\mathrm{G}, 72 \mathrm{~h}$ following the first transfection, Lin $28 \mathrm{mRNA}$ was reduced by $97 \%$, and Lin 28 protein reduced by $90 \%$.

\section{DISCUSSION}

We have described a new transfection method that is highly effective at inducing gene silencing of two endogenous

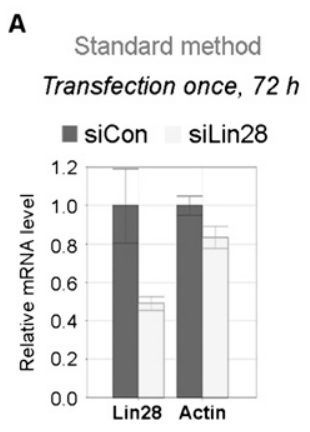

B $\mathrm{H} 1$

Transfection once, $24 \mathrm{~h}$

m siCon $\square$ siLin28
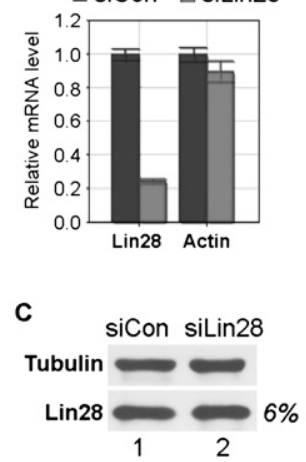

D $\mathrm{H} 1$

$\begin{array}{ll}\text { H1 } & \text { F iPSC } \\ \text { Transfection twice, } 72 h & \text { Transfection twice, } 72 h\end{array}$
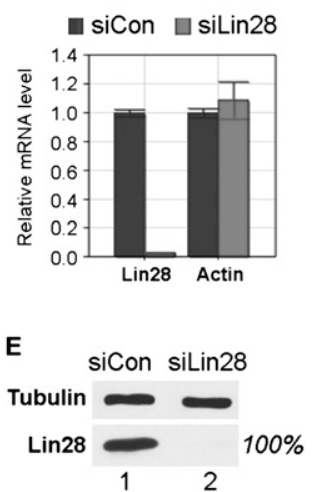

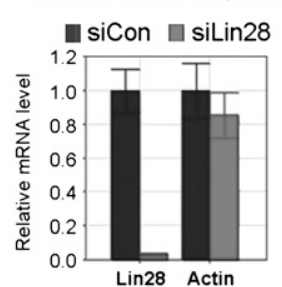

G

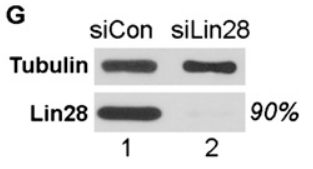

FIGURE 3. siRNA knockdown of Lin 28 in $\mathrm{H} 1(A-E)$ and iPSC $(F, G)$ cells, using standard $(A)$ or new $(B-G)$ method. $(A, B, D, F)$ Results of qRTPCR. Relative mRNA levels are shown after normalization against $\beta$-tubulin mRNA. mRNA levels in cells transfected with siCon were arbitrarily set as 1 . Numbers are mean $\pm \operatorname{SD}(n=3)$. $(C, E, G)$ Representative Western blot results. Antibodies used are shown on the left. Protein bands on Western blots were determined using Bio-Rad Quantity One software, and calculated after normalization against $\beta$-tubulin loading control. Lin28 protein levels in cells transfected with siCon were arbitrarily set as $100 \%$. Percentages at right indicate knockdown efficiencies. 
A $\mathrm{H} 1$

Transfect once, $24 \mathrm{~h}$

siCon $\square$ siOct4

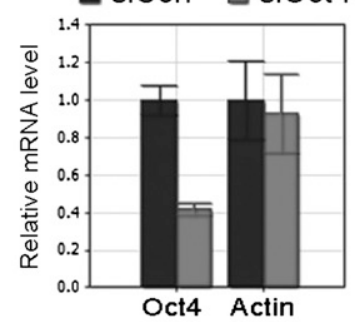

B

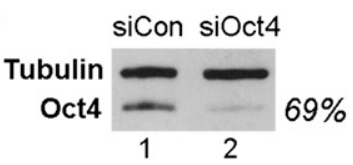

C $\mathrm{H} 1$

Transfect twice, $72 \mathrm{~h}$
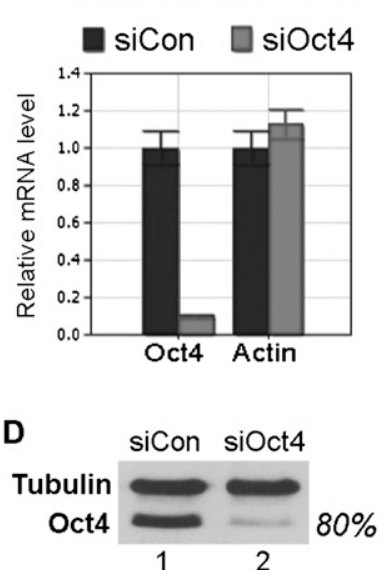

E H1

Transfection twice, $6 d$

siCon $\square$ siOct4

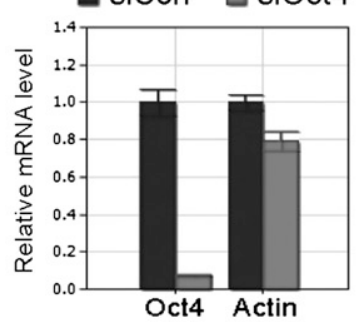

F

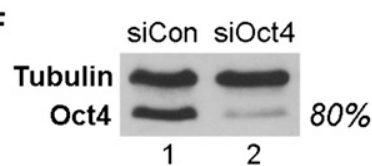

G $H 7$ Transfection twice, $72 h$ siCon $\square$ siOct4
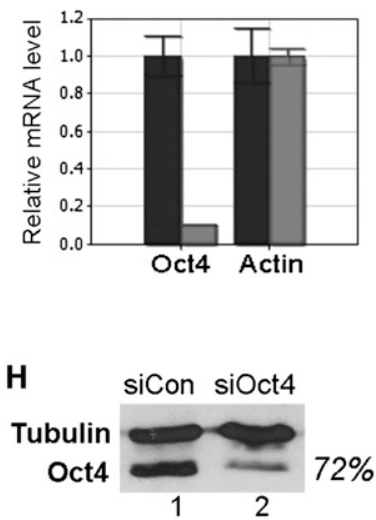

FIGURE 4. siRNA knockdown of Oct4 in $\mathrm{H} 1$ and $\mathrm{H} 7$ cells. $(A, C, E, G)$ Results of qRT-PCR. Relative mRNA levels are shown after normalization against $\beta$-tubulin mRNA. mRNA levels in cells transfected with siCon were arbitrarily set as 1 . Numbers are mean $\pm \mathrm{SD}(n=3)$. $(B, D, F, H)$ Representative Western blot results. Antibodies used are shown on the left. Oct4 protein levels in cells transfected with siCon were arbitrarily set as $100 \%$. $(A-F)$ Results from $\mathrm{H} 1$ transfection. $(G, H)$ results from $\mathrm{H} 7$ transfection.

pluripotency factors in two hESC lines and one iPSC line. While comparably efficient to the other delivery methods (Zaehres et al. 2005; Hohenstein et al. 2008), our method is simpler and far less expensive. The method should allow for fast and convenient evaluation of silencing of genes involved in self-renewal and survival of hESCs. In general, the maximal knock-down of the target genes is achieved within the first 24-72 h, with morphological changes also evident within this time frame. The knock-down effect is lessened afterward, but the initial effect can be effective enough, in the case of certain genes, to drive hESC differentiation. This is apparently the case for the Oct4 knock-down. The persisting low-level expression of Oct4 for at least $6 \mathrm{~d}$ (Fig. $4 \mathrm{E}, \mathrm{F})$ likely reflects the fact that the initial knock-down of Oct4 expression was sufficient to drive hESC differentiation. The differentiated hESCs then, in turn, down-regulates Oct4 expression. In addition, we show that two consecutive siRNA transfections at a 48 -h interval were well tolerated by the hESCs (Figs. 3D-G, 4C-H). Thus, additional rounds of siRNA transfection may extend the knockdown period of time even further, in case it is necessary for observing effects of other genes involved in hESC self-renewal/differentiation. Our method will be equally useful in understanding mechanisms of cell de-differentiation, as well as the epigenetics behind iPSCs, thereby facilitating the clinical potential of iPSCs.

As evident from the above analysis, transient gene knockdown is very useful in addressing gene function: The transient gene knockdown as the method presented here is quick and less consuming, allows tracking the direct gene partner and effect, and is useful especially for those genes crucial for cell survival; the constitutive gene knockdown needs to put in more effort and is an important tool for addressing long-term effect, signaling networks, and longterm physiological aspects.

So far, we have found a ratio of 9-36 pmol of siRNA (or $0.4 \mu \mathrm{g}$ of plasmid DNA) per $5 \mu \mathrm{L}$ of Lipofactamine and a ratio of $1 \mu \mathrm{L}$ of transfection solution per $15 \mu \mathrm{L}$ of fresh growth medium to work well in obtaining $>90 \%$ genesilencing efficiencies using both siLin 28 and siOct 4 (or 70\% plasmid DNA transfection efficiency). However, these
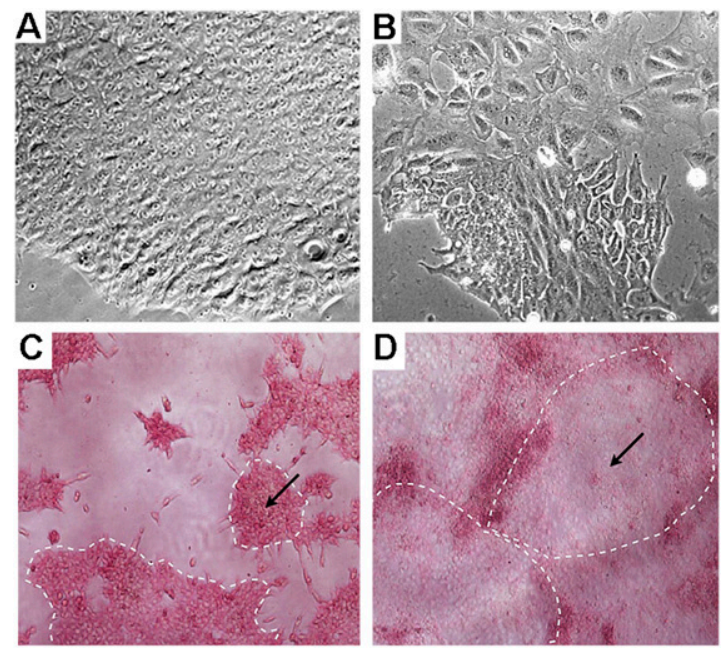

FIGURE 5. Effects of Oct4 knockdown on cell differentiation. H1 cells were transfected with siCon $(A, C)$ or siOct4 $(B, D)$. Cells were analyzed by phase contrast microscopy $(A, B, 200 \times$ magnification) or stained for alkaline phosphatase expression $(C, D, 40 \times$ magnification). $(C, D)$ Cell colonies were outlined with arrows pointing to the center of the colonies. As differentiated cells had enlarged cell size and increased cytoplasmic area, the colonies thus appeared larger and fused together with each other. 
ratios may be adjusted based on siRNA/plasmid DNA, cell type, and culture condition to achieve maximum transfection efficiency, while avoiding off-target effects. We have also found that increasing the ratio between the transfection solution and fresh growth medium (for example, $1 \mu \mathrm{L}$ of transfection solution per $4 \mu \mathrm{L}$ of fresh growth medium) tends to increase transfection efficiency, which is sometimes compromised by increased cytotoxicity (data not shown). We have noticed that siRNA knockdown efficiencies vary between different siRNAs, as well as target genes using our method. Although a single round of transfection of siLin 28 is often sufficient to inhibit Lin 28 expression by $>90 \%$, additional rounds of siRNA transfection are necessary for some other siRNAs, including siOct4 (data not shown).

The method described here is equally useful for both plasmid DNA and siRNA transfection into hESCs and iPSCs. In addition, this method can be applied to other hard-to-transfect cells, including primary cells, except that ROCK inhibitors will not be necessary and Accutase will be replaced by trypsin (Zhang et al. 2007). Finally, the protocol described here that enables the dramatic increase in the efficiency of single cell cloning should be of great value for the studies involved in clonal selection in hESCs and iPSCs.

\section{MATERIALS AND METHODS}

\section{Cell culture}

The hESC lines $\mathrm{H} 1$ and $\mathrm{H} 7$ (listed in the NIH hESC registry under the names of WA01 and WA07, respectively) were obtained from WiCell. The iPSC line was generated at the Yale Stem Cell Center using Yamanaka factors (Takahashi et al. 2007). The cells were cultured in an undifferentiated state in Matrigel (BD Pharmingen CA)-coated plates under feeder-free and defined component conditions (Yao et al. 2006) at $37^{\circ} \mathrm{C}$ in hESC culture incubators (5\% $\mathrm{CO}_{2}, 5 \% \mathrm{O}_{2}$, and $90 \%-95 \%$ humidity). Cell growth media were composed of Dulbecco's modified Eagle medium (DMEM)/ F12 (Invitrogen), supplemented with 1\% MEM-nonessential amino acids (Invitrogen), $1 \mathrm{mM} \mathrm{L}$-glutamine, $1 \%$ penicillinstreptomycin $(\mathrm{P} / \mathrm{S}), 50 \mathrm{ng} / \mathrm{mL}$ basic fibroblast growth factor (Millipore), N2 supplements $(1 \times)$, and B27 supplements $(1 \times)$ (Invitrogen). Cells were maintained with daily medium change and passaged weekly by dissociation into small clumps using $1 \mathrm{mg} / \mathrm{mL}$ dispase (StemCell Technology). The hESCs and iPSCs used were between passages 30 and 60 and 10 and 15 , respectively. Free of mycoplasma contamination, the cells exhibited normal karyotype, expressed common ES markers, and were able to differentiate into the three germ layers (data not shown).

\section{siRNAs, antibodies, and plasmids}

siLin28 (ON-TARGETplus SMARTpool), siOct4 (ON-TARGETplus SMARTpool), and siCon were purchased from Dharmacon. Antibodies used in the study were anti-Lin28 (Abcam, ab46020), anti$\beta$-tubulin (Abcam, ab6046), and anti-Oct4 (Chemicon, AB3209). Plasmid pSUPER-GFP (5.4 kb, driven by a CMV promoter, Oligoangine) was a gift from Dr. Katherine Uyhazi.

\section{Cloning assay}

On the day of cloning, healthy and exponentially growing cells (60\%-70\% confluence on day 5 after passaging) were fed $6-8 \mathrm{~h}$ prior to transfection, followed by incubation in the presence of ROCK Inhibitor Y-27632 (Calbiochem) (working concentration at $10 \mu \mathrm{M}$ in ES growth medium) for $1 \mathrm{~h}$. These steps were found to be necessary to preserve cell viability after transfection. Cells were then dissociated into single-cell suspension using Accutase (STEMCELL) and pelleted by centrifugation. Cell pellets containing $1 \times 10^{5}, 1 \times 10^{4}$, or $1 \times 10^{3}$ cells were each resuspended in $2 \mathrm{~mL}$ of ES growth medium and plated onto one well of a Matrigel-coated six-well plate. Colonies were counted $14 \mathrm{~d}$ after plating, and cloning efficiencies calculated.

\section{siRNA and plasmid DNA transfection}

A cell pellet containing $1 \times 10^{6}$ cells produced by the abovedescribed method was resuspended in $100 \mu \mathrm{L}$ of siRNA/lipid or plasmid DNA/lipid solution (see below), and the suspension incubated at room temperature for 10-15 min (note, incubation $>15$ min will decrease cell viability). At the end of incubation, $1.5 \mathrm{~mL}$ of prewarmed fresh growth medium (containing ROCK inhibitor at a final concentration of $10 \mu \mathrm{M}$ ) was added and the suspension transferred into a Matrigel-coated well of a six-well plate, followed by incubation in a hESC incubator overnight. The medium was replaced with fresh growth medium the second day of the transfection. In the case of double transfection, the cells were transfected the second time $48 \mathrm{~h}$ following the first transfection using the same protocol as described above.

To prepare siRNA/lipid solutions for each well of a six-well plate, 9 or $36 \mathrm{pmol}$ of siRNAs were diluted in $50 \mu \mathrm{L}$ of OPTIMEMI (Invitrogen) and incubated at room temperature for $5 \mathrm{~min}$. In a separate tube, $5 \mu \mathrm{L}$ of Lipofectamine 2000 (Invitrogen) was diluted in $50 \mu \mathrm{L}$ of OPTI-MEMI and incubation carried out for $5 \mathrm{~min}$ at room temperature. The contents of the two tubes were combined by gentle pipetting and incubated at room temperature for $30-50 \mathrm{~min}$. The resulting $100 \mu \mathrm{L}$ of transfection solution was used to resuspend the cell pellet $\left(1 \times 10^{6}\right)$ produced after Accutase dissociation (see above).

For siRNA transfection using the standard method, cells were seeded in a 6 -well plate $5 \mathrm{~d}$ prior to transfection. On the day of transfection, $100 \mu \mathrm{L}$ of siRNA/lipid solution diluted in $1.5 \mathrm{~mL}$ of growth medium was added to each well of cells grown as singlelayer colonies attached to the plate. ROCK inhibitors were not involved before, during, or after the transfection. RNAs were extracted for analysis $72 \mathrm{~h}$ after the transfection.

The protocols were the same for the transfection of plasmid DNA, except that for each well of a six-well plate transfection, 1.6 $\mu \mathrm{g}$ of a reporter plasmid instead of siRNA was used to make the transfection solution.

\section{RNA extraction, qRT-PCR, protein extraction, and Western blot analyses}

These were done as previously described (Xu and Huang 2009). The PCR primers specific for the human genes are: $\beta$-actin forward: $5^{\prime}$-ATCAAGATCATTGCTCCTCCTGAG; $\beta$-actin reverse: $5^{\prime}$-CTG CTTGCTGATCCACATCTG; tubulin forward: $5^{\prime}$-CGTGTTCGGC CAGAGTGGTGC, tubulin reverse: 5'-GGGTGAGGGCATGACGC 
TGAA; Lin28 forward: 5'-CGGGCATCTGTAAGTGGTTC, Lin28 reverse: 5' -CAGACCCTTGGCTGACTTCT; Oct4 forward: 5' -GTG GAGGAAGCTGACAACAA, Oct4 reverse: 5' - GCCGGTTACAGA ACCACACT.

\section{Alkaline phosphatase staining}

Cells were stained using Leukocyte Alkaline phosphatase kit (Fisher) according to the manufacturer's protocols.

\section{ACKNOWLEDGMENTS}

We thank Diane Krause for critical reading of the manuscript, Zheng Wang for helping with AP staining, and Katerine Uyhazi and Octavian Henegariu for plasmids. This material is based on work supported by the State of Connecticut under the Connecticut Stem Cell Research Grants Program (09SCAYALE14 to Y.H., 06SCD01 and 08SCD04 to H.L.). Its contents are solely the responsibility of the authors and do not necessarily represent the official views of the State of Connecticut, the Department of Public Health of the State of Connecticut, or Connecticut Innovations, Inc.

Received July 4, 2010; accepted September 16, 2010.

\section{REFERENCES}

Bajpai R, Lesperance J, Kim M, Terskikh AV. 2008. Efficient propagation of single cells Accutase-dissociated human embryonic stem cells. Mol Reprod Dev 75: 818-827.

Claassen DA, Desler MM, Rizzino A. 2009. ROCK inhibition enhances the recovery and growth of cryopreserved human embryonic stem cells and human induced pluripotent stem cells. Mol Reprod Dev 76: $722-732$.
Hohenstein KA, Pyle AD, Chern JY, Lock LF, Donovan PJ. 2008. Nucleofection mediates high-efficiency stable gene knockdown and transgene expression in human embryonic stem cells. Stem Cells 26: 1436-1443.

Pei D. 2009. Regulation of pluripotency and reprogramming by transcription factors. J Biol Chem 284: 3365-3369.

Qiu C, Ma Y, Wang J, Peng S, Huang Y. 2010. Lin28-mediated posttranscriptional regulation of Oct4 expression in human embryonic stem cells. Nucleic Acids Res 38: 1240-1248.

Takahashi K, Tanabe K, Ohnuki M, Narita M, Ichisaka T, Tomoda K, Yamanaka S. 2007. Induction of pluripotent stem cells from adult human fibroblasts by defined factors. Cell 131: 861872.

Thomson JA, Itskovitz-Eldor J, Shapiro SS, Waknitz MA, Swiergiel JJ, Marshall VS, Jones JM. 1998. Embryonic stem cell lines derived from human blastocysts. Science 282: 1145-1147.

Watanabe K, Ueno M, Kamiya D, Nishiyama A, Matsumura M, Wataya T, Takahashi JB, Nishikawa S, Muguruma K, Sasai Y. 2007. A ROCK inhibitor permits survival of dissociated human embryonic stem cells. Nat Biotechnol 25: 681-686.

Xu B, Huang Y. 2009. Histone H2a mRNA interacts with Lin28 and contains a Lin28-dependent posttranscriptional regulatory element. Nucleic Acids Res 37: 4256-4263.

Yao S, Chen S, Clark J, Hao E, Beattie GM, Hayek A, Ding S. 2006. Long-term self-renewal and directed differentiation of human embryonic stem cells in chemically defined conditions. Proc Natl Acad Sci 103: 6907-6912.

Yu J, Vodyanik MA, Smuga-Otto K, Antosiewicz-Bourget J, Frane JL, Tian S, Nie J, Jonsdottir GA, Ruotti V, Stewart R, et al. 2007. Induced pluripotent stem cell lines derived from human somatic cells. Science 318: 1917-1920.

Zaehres H, Lensch MW, Daheron L, Stewart SA, Itskovitz-Eldor J, Daley GQ. 2005. High-efficiency RNA interference in human embryonic stem cells. Stem Cells 23: 299-305.

Zhang M, Guller S, Huang Y. 2007. Method to enhance transfection efficiency of cell lines and placental fibroblasts. Placenta 28: 779 782. 

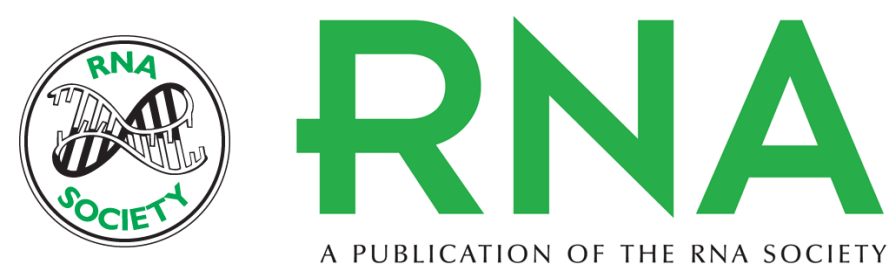

A PUBLICATION OF THE RNA SOCIETY

\title{
High-efficiency siRNA-based gene knockdown in human embryonic stem cells
}

Yinghong Ma, Jianyu Jin, Chunsheng Dong, et al.

RNA 2010 16: 2564-2569 originally published online October 26, 2010

Access the most recent version at doi:10.1261/rna.2350710

\author{
References This article cites 13 articles, 4 of which can be accessed free at: \\ http://rnajournal.cshlp.org/content/16/12/2564.full.html\#ref-list-1 \\ License
} Email Alerting $\begin{aligned} & \text { Receive free email alerts when new articles cite this article - sign up in the box at the } \\ & \text { Service }\end{aligned}$ top right corner of the article or click here. 